CASE REPORT

\title{
ALK positive inflammatory myofibroblastic tumour of the pineal region
}

\author{
A J Clarke, T S Jacques, M J Galloway, M Thom, N D Kitchen, G T Plant
}

J Clin Pathol 2005;58:981-983. doi: 10.1136/icp.2004.022947

Inflammatory myofibroblastic tumours (IMTs) are an uncommon spindle cell neoplasm with a dense inflammatory infiltrate, usually encountered in children. IMTs of the central nervous system are extremely rare. This report describes the case of an IMT in a 61 year old man, in the pineal region. The tumour was completely excised, and immunohistochemistry demonstrated anaplastic lymphoma kinase 1 expression. There was no tumour recurrence during 18 months of follow up. Our case extends both the age range and sites of occurrence of this rare tumour.

I: flammatory myofibroblastic tumours (IMTs) are distinctive lesions composed of myofibroblastic spindle cells with a prominent inflammatory infiltrate of lymphocytes, macrophages, and granulocytes. These tumours are encountered throughout the body, but are most frequently found in children, in the lungs or in relation to the gastrointestinal or genitourinary systems. Involvement of the central nervous system is very rare, with only around 60 cases reported in the literature. Recently, a subset of IMTs has been identified that overexpresses the tyrosine kinase anaplastic lymphoma kinase (ALK) as a result of clonal cytogenetic rearrangements. We report the case of an ALK positive IMT arising from the third ventricle, which was completely resected. The tumour was from a patient of an older age group than has previously been described, either within or outside of the central nervous system.

\begin{abstract}
"Inflammatory myofibroblastic tumours are encountered throughout the body, but are most frequently found in children, in the lungs or in relation to the gastrointestinal or genitourinary systems"
\end{abstract}

\section{CASE REPORT \\ Clinical presentation and imaging}

A 61 year old man with no relevant past medical history was referred because of bilateral optic disc swelling. The finding was an incidental discovery during an eye examination. There had been no preceding symptoms, features of focal neurological deficit, or raised intracranial pressure. Magnetic resonance imaging (fig 1 ) revealed a $2 \mathrm{~cm}$ mass within the third ventricle, arising from the region of the pineal gland. It returned a heterogeneous signal on $\mathrm{Tl}$ and $\mathrm{T} 2$ weighted sequences, and was enhanced after the administration of gadolinium. There was associated mild hydrocephalus and oedema of the surrounding parenchyma. On the imaging appearances, resection was planned.

\section{Surgery and postoperative course}

The tumour was approached from a transventricular route and found to be a $2 \mathrm{~cm}$ mass of fleshy character and of moderate vascularity, apparently arising directly from the pineal gland. It was completely removed without immediate complication. Postoperatively, the patient recovered from a temporary amnesic syndrome, and no tumour recurrence was evident during the 18 months of follow up.

\section{HISTOPATHOLOGICAL EXAMINATION}

Histology (fig 2) showed a well demarcated cellular lesion surrounded by brain parenchyma and compressed choroid plexus. The lesion consisted of areas of dense lymphocyte and plasma cell infiltrates, with intervening regions composed of elongated fibroblast-like spindle cells and large histiocytic cells with granular cytoplasm, which had a dense vascular network and a loose myxoid stroma. There was mild nuclear pleomorphism and rare mitotic figures. The spindle cells showed positive immunostaining for vimentin and desmin, and focal weak staining for epithelial membrane antigen. They also showed strong cytoplasmic immunoreactivity for ALK (ALK-1 antibody). Immunostaining for smooth muscle actin was negative. The lymphocytic infiltrate was composed of a mixture of $\mathrm{CD} 3$ positive T cells and CD20 positive B cells. Immunoglobulin light chain staining showed a polytypic pattern. Immunohistochemistry for CD68 confirmed the large granular cells to be of histiocyte/macrophage lineage. No positive immunostaining was seen for CDla or S100, arguing strongly against a diagnosis of Langerhan's cell histiocytosis or Rosai-Dorfman syndrome, respectively. The possibility of an underlying carcinoma or germ cell tumour was excluded by the absence of immunostaining for cytokeratin (MNF116 antibody) and placental alkaline phosphatase, respectively. No acid fast bacilli were identified with Ziehl-Neelson staining.

\section{DISCUSSION}

The distinctive histological features seen in our case are typical of an inflammatory myofibroblastic tumour and are characterised by spindle shaped myofibroblasts and a patchy chronic inflammatory cell infiltrate rich in plasma cells and lymphocytes. IMTs remain a rare tumour, the pathogenesis of which is only now beginning to be understood.

These tumours were originally described after lung infections, ${ }^{1}$ and were thought to be reactive and benign. However, some of these tumours behave malignantly. Cytogenetic rearrangements within the short arm of chromosome 2 were shown to fall within a $100 \mathrm{~kb}$ region containing the tyrosine kinase ALK. ${ }^{2}$ ALK was originally identified in anaplastic large cell lymphoma, where it is part of a hybrid fusion protein formed by translocation, usually with nucleophosmin (NPMALK). ${ }^{3}$ It is clear that ALK expression in anaplastic lymphoma is associated with a good prognosis, ${ }^{4}$ but in IMT ALK is associated with young age and perhaps poor

Abbreviations: ALK, anaplastic lymphoma kinase; IMT, anaplastic lymphoma kinase 


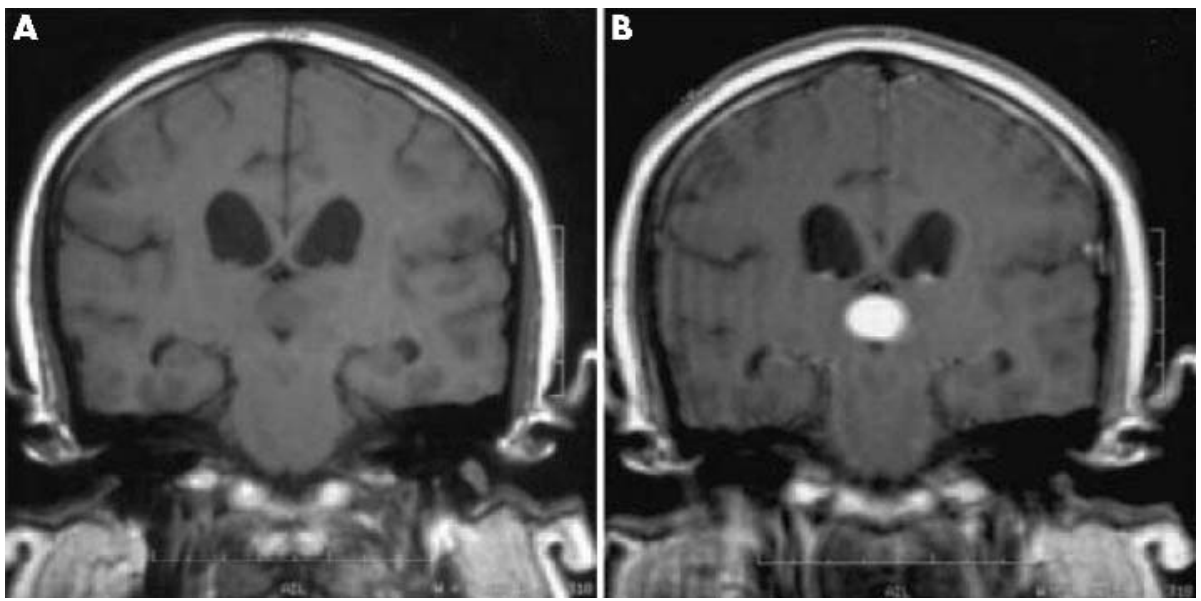

Figure 1 Coronal $\mathrm{Tl}$ weighted magnetic resonance images $(A)$ before and (B) after contrast with gadolinium showing an enhancing $2 \mathrm{~cm}$ tumour in the third ventricle in the region of the pineal gland, with mild hydrocephalus.

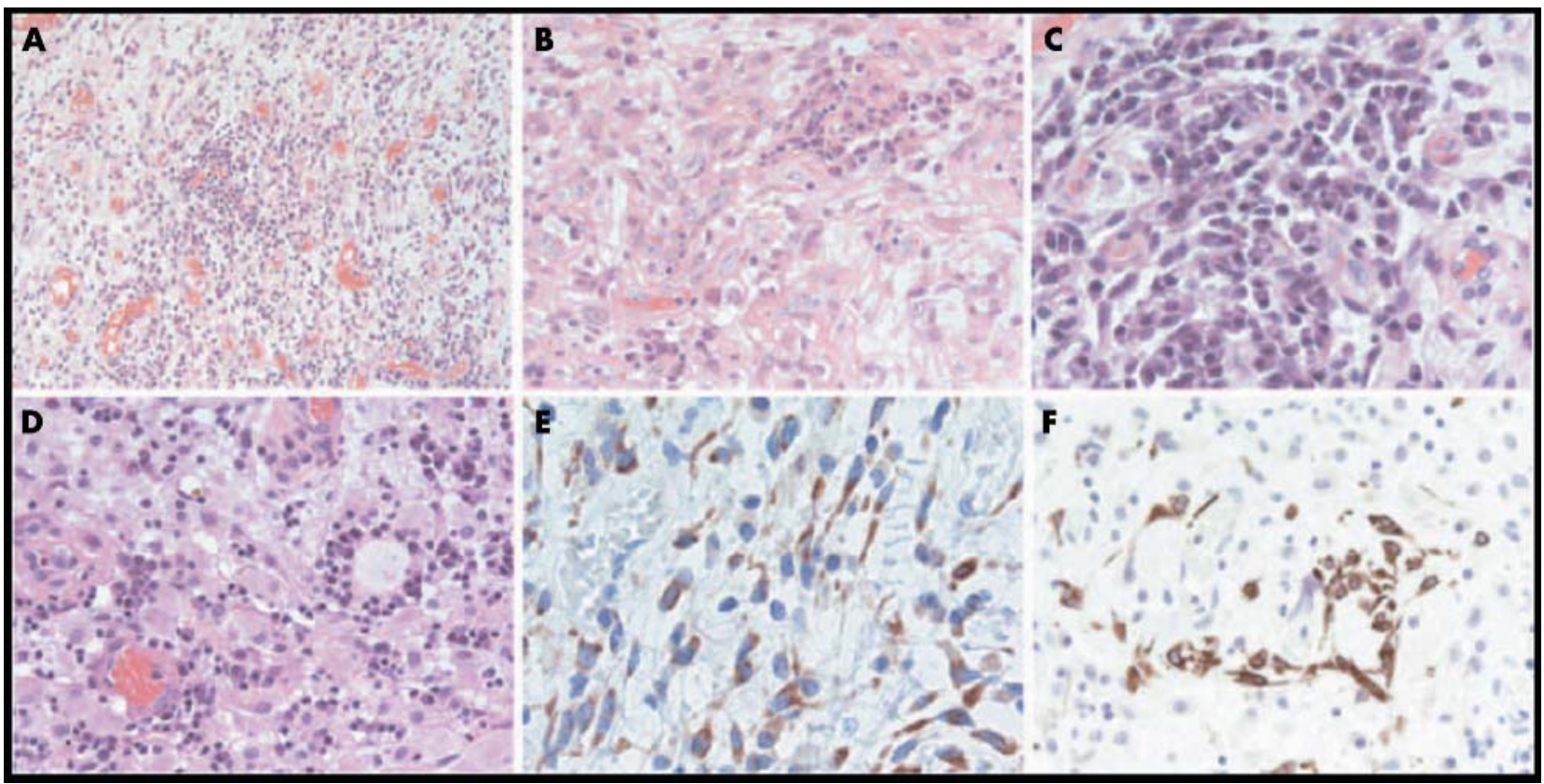

Figure 2 (A, B) Histology showed regions composed of elongated fibroblast-like spindle cells, mixed with (A, D) dense lymphocyte and plasma cell infiltrates. (C) There were intervening areas with large histiocytic cells with granular cytoplasm. The spindle cells showed positive immunostaining for (E) anaplastic lymphoma kinase 1 and (F) desmin.

prognosis. ${ }^{5}$ Only two cases of ALK expression in central nervous system IMT have been described, both of which displayed an aggressive course and were seen in young patients. ${ }^{6-8}$

\section{Take home messages}

- We describe an extremely rare case of inflammatory myofibroblastic tumour of the central nervous system in a 61 year old man

- The tumour expressed anaplastic lymphoma kinase 1 and there was no recurrence during 18 months of follow up

- This case extends both the age range and sites of occurrence of this rare tumour
"Inflammatory myofibroblastic tumours remain a rare tumour, the pathogenesis of which is only now beginning to be understood"

IMTs are an increasingly well understood pathological entity, but their rare occurrence within the central nervous system has meant that this subset is less well characterised. We describe a case with ALK overexpression, in a patient from an age group in which IMTs have not been encountered, in a previously unreported location, and with no recurrence during follow up.

\section{Authors' affiliations}

A J Clarke, G T Plant, Department of Neurology, National Hospital for Neurology and Neurosurgery, Queen Square, London WCIN 3BG, UK T S Jacques, M J Galloway, M Thom, Department of Neuropathology, National Hospital for Neurology and Neurosurgery

N D Kitchen, Department of Neurosurgery, National Hospital for Neurology and Neurosurgery 
The patient gave his informed consent for this case to be reported.

Correspondence to: Dr A J Clarke, Department of Neurology, National Hospital for Neurology and Neurosurgery, Queen Square, London WC1N 3BG, UK; alex@actualmedia.com

Accepted for publication 1 November 2004

\section{REFERENCES}

1 Umiker WO, Iverson L. Postinflammatory 'tumour' of the lung. Report of four cases simulating xanthoma, fibroma or plasma cell granuloma. J Thorac Cardiovasc Surg 1954;28:55-63.

2 Griffin CA, Hawkins AL, Dvorak C, et al. Recurrent involvement of 2 p23 in inflammatory myofibroblastic tumours. Cancer Res 1999;59:2776-80.
3 Pulford K, Wade Morris S, York Mason D. Anaplastic lymphoma kinase proteins and malignancy. Curr Opin Hematol 2001;81:231-6.

4 Ten Berge RL, Oudejans JJ, Ossenkoppele GJ, et al. ALK expression in extranodal anaplastic large cell lymphoma favors systemic disease with (primary) nodal involvement and a good prognosis and occurs before dissemination. J Clin Pathol 2000;53:445-50.

5 Coffin CM, Patel A, Perkins S, et al. ALK1 and p80 expression and chromosomal rearrangements involving 2 p23 in inflammatory myofibroblastic tumor. Mod Pathol 2001;14:569-75.

6 Hausler M, Schaade L, Ramaekers VT, et al. Inflammatory pseudotumors of the central nervous system: report of three cases and a literature review. Hum Pathol 2003;34:253-62.

7 Lacoste-Collin L, Roux F, Gomez-Brouchet A, et al. Inflammatory myofibroblastic tumour: a spinal case with aggressive clinical course and ALK overexpression. J Neurosurg 2003;98:218-21.

8 Despeyroux-Ewers M, Catalaa II, Collin L, et al. Inflammatory myofibroblastic tumour of the spinal cord: case report and review of the literature. Neuroradiology 2003;45:812-17. 